\title{
Das Gesundheitswesen im Aargau im 19. Jahrhundert
}

\author{
Von Heinrich Staehelin
}

Das Thema «Gesundheitswesen im Aargau im 19. Jahrhundert» dürfte niemandem grundlegend Neues bieten, der - etwa aus der Lektüre von Jeremias Gotthelf - mit dem Denken und Fühlen des Landvolkes von anno dazumal vertraut ist. Der Aargau war und blieb in dieser Zeit ein Agrarkanton, wenn auch die Industrie darin eine recht bedeutende Rolle spielte. 1837 lebten nur 10\%, 1880 etwa 13,5\% seiner Bevölkerung in Städten, die erst noch diesen Namen nicht einmal richtig verdienten. Die größte Stadt war Aarau mit gut 4000 Einwohnern im Jahre 1837 und knapp $6000 \mathrm{im}$ Jahre 1880. 1888 waren von den fast 200000 Kantonseinwohnern kaum 15000 in den Fabriken, über 80000 aber in der Landwirtschaft tätig. Erschreckend viele dieser Leute waren mittellos. Um die Jahrhundertmitte bezog ein Achtel, später noch immer ein Zwölftel der Gesamtbevölkerung Armenunterstützung, und mindestens ebenso viele Menschen waren der Armut bedenklich nahe ${ }^{1}$.

Für eine zeitgemäße, wissenschaftlich orientierte Medizin, die natürlich auch damals ihr teures Geld kostete, hatte dieses Volk wenig übrig - nicht nur, weil es nicht zahlen konnte oder wollte, sondern wohl noch mehr, weil es an seinen alten Bräuchen hing und Neuerungen mißtraute, besonders wenn sie von oben und aus der Stadt kamen. Als 1836 und 1837 die rote Ruhr (eine mit blutigem Stuhlgang verbundene Krankheit des Unterleibs) grassierte, zogen gegen $90 \%$ aller Erkrankten, darunter auch bemittelte, den Arzt überhaupt nicht oder erst im äußersten Notfall zu und behalfen sich mit allen möglichen Hausmittelchen - in diesem Fall mit Käse, Rotwein und kaltem Wasser ${ }^{2}$. Stöße von ärztlichen Berichten und Gerichtsakten berichten von Quacksalbern aller Art, und die Bemühungen der Behörden, diesen das Handwerk zu legen, fanden durchaus nicht immer Anerkennung im Volke. Später verband sich diese Abwehrhaltung mit dem politischen Widerstand gegen die herrschende liberale Oberschicht in der sogenannten «demokratischen Bewegung» der 1860 er Jahre ${ }^{3}$. Das Finanzreferendum wurde erkämpft und mißbraucht, um die kostspieligen Spitalbauprojekte der Regierung zu sabotieren; man verlangte die «Umgestaltung der Medizinalgesetzgebung in einem freisinnigen Geiste», d.h. in dem Sinne, daß die Ausübung eines Medizinalberufs nicht vom Besitz eines staatlichen Patents abhängig sein sollte, während eine zeitgemäße Reform des in vielen Teilen veralteten Sanitätsgesetzes von 
1836 in der Volksabstimmung scheiterte. So besehen, wirkte sich die Demokratisierung des öffentlichen Lebens, die durchaus auch ihre guten Seiten hatte, eher zum Nachteil der großen Masse aus.

Es ist wohl im allgemeinen, kaum aber im einzelnen festzustellen, an welchen Krankheiten die Menschen von damals hauptsächlich litten. In den amtlichen Berichten wurden, so gut es eben ging, alle Leiden verzeichnet, die sich in größerem Maße auf die Gesellschaft auswirkten - also beispielsweise Epidemien -, aber in einer Terminologie, die der heutigen in weiten Teilen nicht mehr entspricht. Und was soll man zur Bemerkung der Regierung vom Jahr 1865 sagen, daß Lungentuberkulose «in auffallend kleiner Zahl als Todesursache angegeben» wurde $^{4}$ ? Bestimmt war ein sehr hoher Prozentsatz unter den Zehntausenden von Heimarbeitern, die von Kindesbeinen an täglich zwölf Stunden und mehr in lichtarmen, feuchten Kellern ihre Tücher woben, tuberkulös - nur starben offensichtlich die meisten an einer andern, akuten Krankheit. - Neben Unfällen, Apoplexien usw. herrschten akute Infektionskrankheiten als Todesursachen vor ${ }^{5}$. Bis gegen 1860 wirkten sich epidemisch auftretende Unterleibskrankheiten am verheerendsten aus: so 1836 und 1837 die rote Ruhr, 1854 die Cholera, immer wieder auch der Typhus. Später überwogen Krankheiten der Atemwege Keuchhusten, Bronchialkatarrhe, Lungenentzündungen - sowie Kinderkrankheiten wie Scharlach und Masern. Gelegentlich werden Fälle von Krebs erwähnt. Häufig waren auch Mangelkrankheiten, Mißwuchs, chronische Leiden der Sinnesorgane usw.; solcher Gebrechen wegen war noch um 1880 ein Drittel der Stellungspflichtigen militärdienstuntauglich.

Neben den noch lange Zeit geradezu unmenschlichen Arbeitsbedingungen namentlich für Heim- und Fabrikarbeiter gaben falsche und unzureichende Ernährung und mangelnde Hygiene einen nur zu guten Boden für alle möglichen Krankheiten ab. Das bedeutet aber auch, daß der Gesundheitszustand der unteren Gesellschaftsschichten erheblich schlechter war als derjenige der höheren Zeugnisse mancher Ärzte bestätigen dies. Seit dem Katastrophenjahr 1816/1817 ist zwar im Aargau kaum jemand mehr buchstäblich verhungert; aber noch immer genügten eine, zwei Mißernten, um die ärmeren Leute bittere Not leiden zu lassen. Bis in die 1850 er Jahre hinein folgte einem Fehljahr unweigerlich ein Anstieg der Sterberate; von 1860 an aber, als fehlende Lebensmittel rasch und billig mit der Eisenbahn beschafft werden konnten, muß sich die mittlere Lebenserwartung im Aargau bedeutend gehoben haben. - Mit den sanitären Zuständen war es noch lange Zeit bös bestellt. Besonders ärmere Leute lebten oft eng zúsammengedrängt in düsteren, ungesunden Wohnungen. Epidemische Unterleibskrankheiten wurden häufig durch verdorbenes Trinkwasser verbreitet. Auch 
die Städte blieben davon nicht verschont: in Aarau, wo man das Trinkwasser größtenteils dem Stadtbach entnahm, der einige Kilometer oberhalb mitten durch das Dorf Suhr fließt, forderte die Cholera 1854 81 Opfer ${ }^{6}$. Immer wieder mußten die Behörden gegen nachlässige oder gewissenlose Leute einschreiten, die verdorbene Lebensmittel verkauften. Unsachgemäße Pflege schutzbedürftiger Personen tat ein übriges; sie ist nach zeitgenössischen Quellen die Hauptursache für die hohe Kindersterblichkeit. Um 1870 starb fast ein Viertel der Säuglinge im ersten Lebensjahr; vom ersten bis zum fünften Altersjahr starb eines von fünfzehn Kindern ${ }^{7}$. - Erwähnt sei schließlich der Mißbrauch geistiger Getränke, besonders von Branntwein, woran zwar wenige unmittelbar zugrunde gingen, der aber nichtsdestoweniger ein sehr ernstes soziales und medizinisches Problem darstellte. Von 65 Männern, die 1880 in die Heilanstalt Königsfelden eingeliefert wurden, litten 17 an Schäden, die auf übermäßigen Alkoholkonsum zurückzuführen waren ${ }^{8}$.

Mit dem zahlenmäßigen Verhältnis der Ärzte zu den Einwohnern ${ }^{9}$ stand es im Aargau damals noch schlechter als heute : 1837 gab es 91 Ärzte, in den folgenden Jahrzehnten etwas mehr, 1880 aber wiederum nur 91, 1900 hundert. Es traf 1837 einen Arzt auf 2009, 1880 aber einen Arzt auf 2180 Einwohner. Die Situation hatte sich also leicht verschlechtert. Dies gilt auch für die Verteilung der Ärzte über das Kantonsgebiet. Von den rund 240 Gemeinden des Kantons hatten 1837 48, 1880 nur 46 mindestens einen Arzt. Stark bevorzugt waren die Städte, wo 1837 43, 188044 Ärzte wohnten, wovon 8 in Aarau und 10 in Baden. Notstandsgebiete waren vor allem der Jura und die dichtbesiedelten südwestlichen Gegenden. Am meisten Einwohner auf einen Arzt kamen im Bezirk Kulm (1837 5100 , 1880 noch immer 3305). Dr. Joseph Fridolin Wieland, Bezirksarzt daselbst, ansäßig in Schöftland, behandelte 1837 nach eigenen Angaben in 6 Wochen über 500 Ruhrkranke, und zur Bekämpfung dieser Epidemie mußten ihm Kollegen von Aarau und Zofingen zu Hilfe kommen ${ }^{10}$. Arzt Hunziker von Oberkulm legte im Februar und März 1868 auf Hausbesuchen Distanzen von 20 Kilometern und mehr pro Tag zurück ${ }^{11}$. Der Beruf des Landarztes war also auch damals anstrengend und kaum sehr einträglich. - Auch die ärztlichen Gehilfen, Nachfahren der alten Bader und Zahnbrecher, die laut Sanitätsgesetz Zähne ziehen, zur Ader lassen, Blutegel ansetzen und Wunden verbinden durften, zogen es vor, in den Städten zu wohnen, desgleichen die Apotheker, weshalb den meisten Landärzten die Selbstdispensation (Abgabe von Medikamenten an ihre Patienten) gestattet werden mußte. Nur Hebammen anzustellen war jede Gemeinde von über 500 Einwohnern verpflichtet ${ }^{12}$.

Unser Bild vom aargauischen Gesundheitswesen wäre jedoch unvollständig, 
wenn wir nicht noch die Bemühungen von Ärzten, Behörden und anderen führenden Köpfen zu dessen Hebung erwähnten. Auch ohne moderne bakteriologische Kenntnisse war die ärztliche Kunst gegen Seuchen nicht machtlos. Schon wirkungsvolle Absperrmaßnahmen vermochten diese lokal zu begrenzen; von Epidemien, die den ganzen Kanton auf einmal befallen hätten, ist aus jener Zeit nichts bekannt. Und zumindest gegen die Pocken kannte man als wirksame Prophylaxe die Schutzimpfung; der Kanton Aargau führte diese bereits 1806 von Staates wegen ein ${ }^{13}$ und erklärte sie bald darauf für obligatorisch. Ärzte, Behörden, aber auch Geistliche und Lehrer kämpften mit Wort und Tat für bessere hygienische Zustände und übten, wenn nötig, auch Druck auf uneinsichtige Gemeinden aus, nach Erlaß eines aargauischen Fabrikgesetzes (1862) auch auf allzu sparsame Fabrikanten, die bessere Einrichtungen zum Schutz der Gesundheit ihrer Arbeiter für überflüssig ansahen. Allmählich besserte es sich nun mit den Friedhöfen, der Trinkwasserversorgung, den Wohn- und Arbeitsverhältnissen. So gar nichts hat also diese oft als unsozial bezeichnete Epoche für das Wohl der Unterschicht doch nicht getan! Auch die Vorschriften über die Ausübung von Medizinalberufen wurden, dem immer höheren Stand der Wissenschaft entsprechend, verschärft. Um 1800 hatte man den Beruf eines Arztes noch fast wie ein Handwerk erlernen können ${ }^{14}$ - vom Apotheker- und Tierarztberuf ganz zu schweigen. Demgegenüber schrieb das Prüfungsreglement des Konkordats über Freizügigkeit des schweizerischen Medizinal-Personals von 186715, dem der Aargau 1871 beitrat, für diese Berufe den Besuch von Hoch- oder höheren Fachschulen vor und verlangte neben praktischen Fertigkeiten auch den Nachweis einer gründlichen naturwissenschaftlichen Bildung. Die Ärzte hatten in diesen Fächern eine propädeutische Prüfung abzulegen; die eigentliche Fachprüfung umfaßte 9 Fächer, darunter Pädiatrie und Psychiatrie.

Endlich bleibt es eine beachtliche Leistung jener Generationen, dem Kanton Aargau, mannigfachen Widerständen auch sogenannt besserer Kreise zum Trotz, zu zeitgemäßen Spitälern verholfen zu haben ${ }^{16}$. In den Räumlichkeiten des früheren Klosters Königsfelden bei Brugg unterhielt der Staat seit dem Anfang des 19. Jahrhunderts eine Krankenanstalt für körperlich wie für geistig-seelisch Leidende. Sie war jedoch zu klein, beständig überfüllt und unzweckmäßig eingerichtet; die Klagen über sie wollten seit den 1830 er Jahren kein Ende nehmen. Erst 1872 aber konnte nach jahrelangen Bemühungen ein den Anforderungen der Zeit entsprechender Neubau wenigstens für die Irrenanstalt in Betrieb genommen werden. Er kostete etwas über 2 Millionen Franken, eine Summe, die vielen Zeitgenossen, besonders der demokratischen Opposition, horrend erschien. Dieser Bau unter anderem gab dem Volke Anlaß, sich 1870 das Finanzreferendum zu 
erkämpfen - und nun war es mit einer neuen Anstalt für körperlich Kranke, die ebenso nötig gewesen wäre, für einige Zeit aus. Eine solche wurde dann 1887, nachdem sich die politischen Verhältnisse gebessert hatten, in Aarau bezogen. Allerdings mußte man an allen Ecken und Enden sparen, da sie nur 1,2 Millionen Franken kosten durfte; so begnügte man sich mit drei Abteilungen (Medizin, Chirurgie und Gebäranstalt), die in recht bescheidenen Pavillons untergebracht wurden. Immerhin war das Grundstück, auf dem die Krankenanstalt erstellt wurde, großzügig bemessen und damit die Möglichkeit gegeben, diese später zu einem leistungsfähigen Großspital auszubauen.

\section{Quellen}

a) ungedruckte

- Protokolle und Akten des Sanitätsrates, Aargauisches Staatsarchiv, Aarau.

- Protokolle und Akten des Großen Rates und des Regierungsrates betreffend die Gründung der kantonalen Heilanstalten, ibid.

- Kassabuch des Arztes Dr. R. Hunziker, Oberkulm, 1868/69, im Besitz von Herrn Dr. F. Knittel, Gränichen.

b) gedruckte

- Rechenschaftsberichte des Kleinen Rates (R Kl R) und des Regierungsrates (RRR), $1837 \mathrm{ff}$.

- Gesetzessammlungen für den eidgenössischen Kanton Aargau, $1803 \mathrm{ff}$.

- Aarg. Staatskalender, $1837 \mathrm{ff}$.

- Schweizerische Statistik (benützt ab 1860).

- 150 Jahre Kanton Aargau im Lichte der Zahlen, 1803-1953, Aarau 1954.

c) Literatur

- diverse Broschüren zu gesundheitspolizeilichen und gesundheitspolitischen Belangem bei den Akten (vgl. oben) und in der Aargauischen Kantonsbibliothek, Aarau.

- Heinrich Staehelin, Carl Feer-Herzog (1820-1880), in: Argovia 87, Aarau 1975; daselbst weitere Literatur.

\section{Anmerkungen}

1 Nach R Kl R/RRR, Schweiz. Statistik, usw.; vgl. Staehelin, Feer-Herzog, S. $57 \mathrm{ff}$.

$2 \quad$ Akten des Sanitätsrates 1836-1838. 
3 Staehelin, Feer-Herzog, S.206f.

4 RRR 1865, S.87.

5 Nach R Kl R/RRR; genauere Angaben ab 1872 in Schweiz. Statistik.

6 Werner Witz, Über die Cholera asiatica im Kanton Aargau anno 1854 (...), Zürich 1943, S.41f.; Staehelin, Feer-Herzog, S.89.

7 Nach den Zahlenangaben in RRR 1867-1875.

8 RRR 1880, S.21.

$9 \quad$ Staatskalender 1837 und 1880.

10 Arzt Wieland, Schöftland, an den Sanitätsrat, 2.10.1837, Akten des Sanitätsrates 1838; Prot. des Sanitätsrates 4.9.1837, etc.

11 Kassabuch des Arztes Dr. Hunziker, Oberkulm.

12 Gesetz, die Organisation des Sanitätswesens betreffend, 15.12.1836, Neue Sammlung der Gesetze und Verordnungen des Kantons Aargau, Aarau (1831 ff.), Bd.2, S. 194 ff.

13 Verbreitung der Schutzblattern, Verordnung vom 16.6.1806, Sammlung der in Kraft bestehenden Kanton Aargauischen Gesetze und Verordnungen, 3. Bd., Aarau 1811, S. $30 \mathrm{ff}$.

14 Vgl. etwa «Erlernung der Heilkunde», Verordnung vom 18.3.1805, Sammlung (vgl. Anm.13), 2. Bd., Aarau 1809, S.30 ff.

15 Gesetzes-Sammlung für den eidgenössischen Kanton Aargau, Fortsetzung der revidierten Ausgabe, Bd. 7, S. $139 \mathrm{ff}$. und $142 \mathrm{ff}$.

16 Staehelin, Feer-Herzog, S. 142, 199-208 und $292 \mathrm{f}$.

\section{Summary}

$90 \%$ of the inhabitants of the Aargau lived during the 19 th century in the country. They were poor and trusted modern medicine not much. There was a lack of trained medical personel. People died mostly from infections diseases, especially of the abdomen, children's diseases, and respiratory diseases, caused by malnutrition, poor hygiene and working conditions. In 1870 the mortality of infants was over $20 \%$.

In spite of a reluctant population the administration obtained some improvements of health conditions. Vaccination was introduced, the medical personel was scientifically trained and modern hospitals were built: 1872 the psychiatric hospital of Königsfelden, 1887 the Kantonsspital Aarau.

Dr. phil. Heinrich Staehelin

Nordstraße 22

5036 Oberentfelden AG 